\title{
Anomaly of the Deep Lateral Orbital Wall in Two Cases
}

\author{
Hirohiko Kakizaki $^{*},{ }^{1}$, Akihiro Ichinose ${ }^{2}$, Yasuhiro Takahashi ${ }^{1}$ and Masayoshi Iwaki ${ }^{1}$
}

\author{
${ }^{I}$ Department of Ophthalmology, Aichi Medical University, Nagakute, Aichi, Japan \\ ${ }^{2}$ Department of Plastic Surgery, Kobe University School of Medicine. Kobe, Hyogo, Japan
}

Keywords: Deep lateral orbital wall, decompression, anomaly, bone defect, cyst, maxillary sinus.

\section{TO THE EDITOR,}

Deep lateral orbital wall decompression is considered the first choice procedure [1] in treating disfiguring proptosis, as well as, compressive optic neuropathy and congestive orbitopathy $[2,3]$. Although detailed normal anatomy of the deep lateral orbital wall has already been reported $[1,4,5]$, there are no reports of anomalies of the wall. We experienced two types of anomalies of the deep lateral orbital wall in patients who underwent deep lateral orbital wall decompression.

The first patient was a 35 year-old woman who showed bilateral orbital inflammation from Graves' orbitopathy, and underwent 2 cycles of steroid pulse therapy $(1$ cycle: methylprednisolone $10 \mathrm{mg} / \mathrm{kg} / \mathrm{day} \times 3$ days). Although the inflammation subsided completely after the therapy, her Hertel exophthalmometry measurements remained $21 \mathrm{~mm}$ OU. Therefore, she hoped to have orbital decompressions to both orbits. We performed bilateral deep lateral orbital decompressions and her final Hertel measurements improved to $14 \mathrm{~mm}$ OD and $13 \mathrm{~mm} \mathrm{OS}$.

She demonstrated bilateral bone defects of the inferior part of the sphenoid door jamb without tumor invasion as shown by preoperative computed tomography (CT) (Fig. 1A). During the operations, the defects were clearly identified and no tumor invasion was found, consistent with CT. (Fig. 1B). The bone defects continued to the inferior orbital fissure, and the orbital fat was herniated into the defect, continuing to the buccal fat.

The other patient was a 40 year-old woman who suffered from Graves' orbitopathy with left orbital inflammation. She also underwent 2 cycles of steroid pulse therapy. The inflammation subsided completely, but her Hertel exophthalmometry measurements remained at $16 \mathrm{~mm} \mathrm{OD}$ and $18 \mathrm{~mm}$ OS. She hoped to have a left orbital decompression. We performed a left deep lateral orbital decompression and her final left Hertel measurement was 15 $\mathrm{mm}$.

She showed a cystic formation with a little fluid collection in the left lower part of the sphenoid door jamb as shown by preoperative CT (Fig. 2A). The cyst was separated

*Address correspondence to this author at the Department of Ophthalmology, Aichi Medical University, Nagakute, Aichi 480-1195, Japan; Tel: +81-56162-3311; Fax: +81-561-63-7255; E-mail: cosme@d1.dion.ne.jp by a thin cortical bone to the middle cranial fossa and it was filled with air without fluid. During the deep lateral orbital wall decompression surgery, the cyst was clearly identified, and the inner wall was lined by thin paranasal sinus mucosa. With enlarging the cyst and following it inferiorly, it was found to continue to the maxillary sinus (Fig. 2B). No osteolytic lesion was found in the operation. The cyst was an expansion of the maxillary sinus.

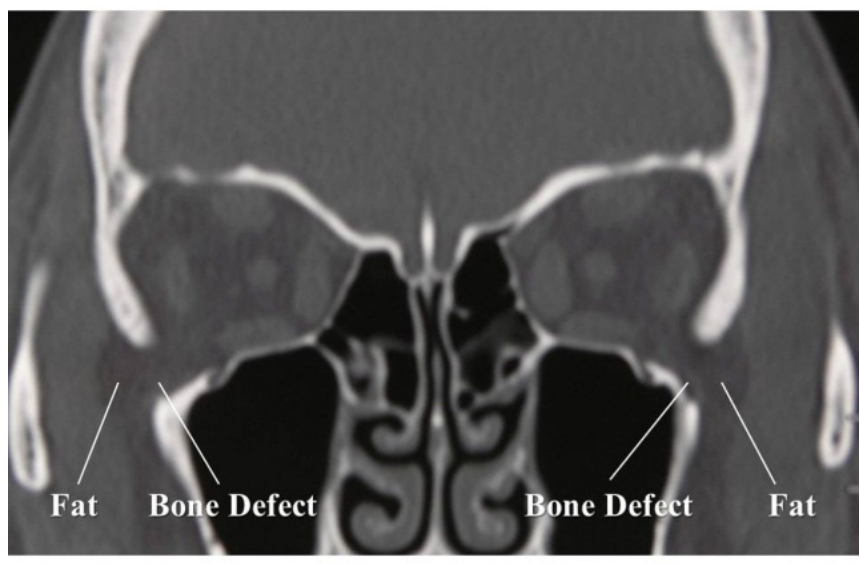

A

Fig. (1A). Bilateral defects of the inferior part of the sphenoid door jamb are shown on computed tomography (CT).

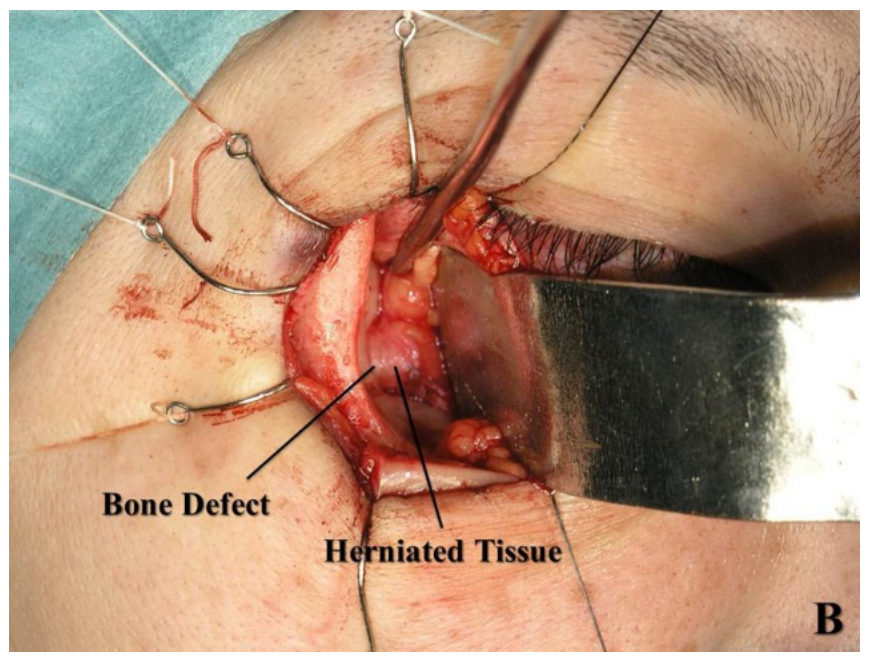

Fig. (1B). A defect is identified during deep lateral orbital wall decompression surgery. 
The first anomaly described above, a defect in the bones, continued to the extraorbital space, and the orbital fat was directly connected with the buccal fat. Therefore, the orbital space was easily enlarged during decompression surgery by removing the buccal fat, and the orbital fat was also easily removed by incising the periosteum around the bone defect.

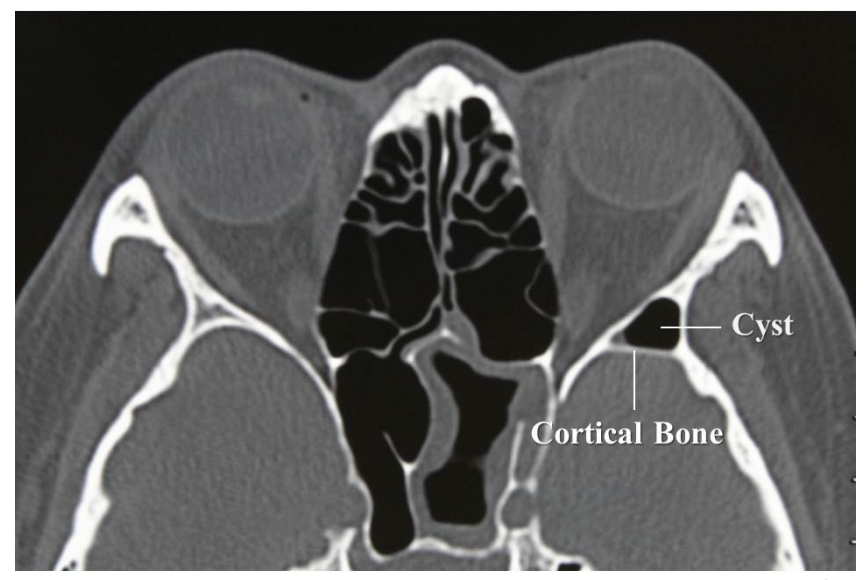

A

Fig. (2A). A cystic formation in the left lower part of the sphenoid door jamb is shown on axial computed tomography (CT).

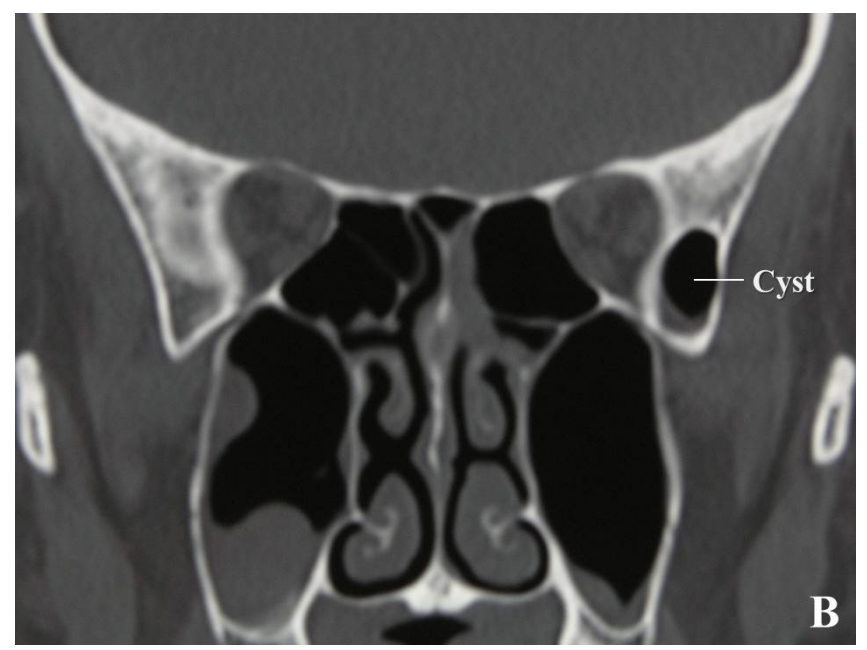

Fig. (2B). Coronal CT is unable to show continuity from the cyst to the maxillary sinus.

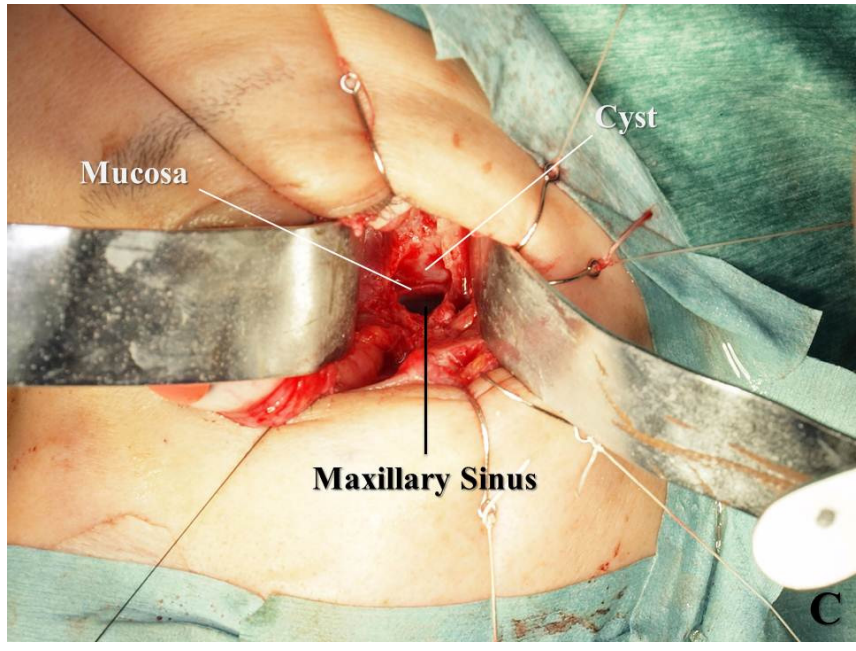

Fig. (2C). The cyst continues to the maxillary sinus.

The second anomaly described above-an expansion of the maxillary sinus - enabled the present surgeon to perform a decompression surgery safely and efficaciously. Since the space was only divided by a thin cortical bone to the middle cranial cavity, maximum bone removal could be achieved by tracing the cortical bone superiorly with bone gliding.

Knowledge of anomalies of the deep lateral orbital wall presented here could be helpful for other surgeons to perform a safe and efficacious deep lateral orbital wall decompression surgery.

\section{REFERENCES:}

[1] Goldberg RA, Kim AJ, Kerivan KM. The lacrimal keyhole, orbital door jamb, and basin of the inferior orbital fissure. Three areas of deep bone in the lateral orbit. Arch Ophthalmol 1998; 116: 161824.

[2] Chang EL, Piva AP. Temporal fossa orbital decompression for treatment of disfiguring thyroid-related orbitopathy. Ophthalmology 2008; 115: 1613-9.

[3] Goldberg RA. The evolving paradigm of orbital decompression surgery. Arch Ophthalmol 1998; 116: 95-6.

[4] Kakizaki H, Nakano T, Asamoto K, Iwaki M. Posterior border of the deep lateral orbital wall-appearance, width, and distance from the orbital rim. Ophthal Plast Reconstr Surg 2008; 24: 262-5.

[5] Kakizaki H, Takahashi Y, Asamoto K, Nakano T, Selva D, Leibovitch I. Anatomy of the superior border of the lateral orbital wall: surgical implications in deep lateral orbital wall decompression surgery. Ophthalmology Plast Reconstr Surg 2011; 27: $60-3$.

(C) Kakizaki et al.; Licensee Bentham Open.

This is an open access article licensed under the terms of the Creative Commons Attribution Non-Commercial License (http: //creativecommons.org/licenses/by$\mathrm{nc} / 3.0 /$ ) which permits unrestricted, non-commercial use, distribution and reproduction in any medium, provided the work is properly cited. 